\title{
Improving the computation of invariants of plane curve singularities
}

\author{
Muhammad Ahsan Binyamin
}

\begin{abstract}
In this article we present an algorithm to compute the incidence matrix of the resolution graph, the total multiplicities, the strict multiplicities and the Milnor number of a reduced plane curve singularity and its implemetation in Singular.
\end{abstract}

\section{Introduction}

In this article we describe how to improve the computation of the resolution graph and related invariants of a reduced plane curve singularity. We were inspired by the use of the computer algebra system SINGULAR( [2],[4]). The SINGULAR libraries hnoether.lib and alexpoly.lib contain procedures to compute the incidence matrix of the resolution graph (for details see section-2 respectively [5],[1]), the multiplicity sequence and other related invariants of a plane curve singularity $(C, 0)$ defined by a polynomial $f \in \mathbb{Q}[x, y]$. If $f$ is not irreducible over $\mathbb{Q}$ then the computation can be very time consuming compared to the computation of the corresponding invariants for the singularities defined by the factors* of $f$ (see section- 4 ). There are combinatorical formulae which relate the invariants of the branches of a curve together with some additional informations, so called contact numbers of the branches (see section-2 for a definition), to the invariants of the curve. Therefore it is much faster to compute the invariants for the branches and some additional invariants and obtain on this basis the invariants of the curve we started with.

Key Words: Resolution Graph, Total and Strict Multiplicities,Hamburger-Noether Expansion.

Received: October, 2011.

Accepted: February, 2012.

* The factors of $f$ may be not analytically irreducible. In this case the splitting to the branches will be computed by the procedures of the SINGULAR-library mentioned above. 


\section{The resolution graph and the multiplicity sequences of a plane curve singularity}

The following definitions and results can be found in [5],[1],[3].

Let $C=\bigcup_{i=1}^{r} C_{i} \subset \mathbb{C}^{2}$ be a small representative of a reducible plane curve singularity with branches $C_{1}, \ldots, C_{r} \quad r \geq 2$. Assume that $X_{i} \stackrel{\pi_{i}}{\rightarrow} \ldots \stackrel{\pi_{2}}{\rightarrow} X_{1} \stackrel{\pi_{1}}{\rightarrow} \mathbb{C}^{2}$ is a sequence of blowing up points. Denote by $E^{(i)}=\left(\pi_{1} \circ \ldots \circ \pi_{i}\right)^{-1}(0)$ the exceptional divisor, $C^{(i)}=\overline{\left(\pi_{1} \circ \ldots \circ \pi_{i}\right)^{-1}(C \backslash\{0\})}$ the strict transform and $\left(\pi_{1} \circ \ldots \circ \pi_{i}\right)^{-1}(C)$ the total transform of C. Let $X_{i+1} \stackrel{\pi_{i+1}}{\rightarrow} X_{i}$ be the blowing up of $X_{i}$ in all points of $C^{(i)} \cap E^{(i)}$ which are still singular on $C^{(i)}$ or nontransversal intersection of $C^{(i)}$ with $E^{(i)}$ that is the points with intersection multiplicity of $C^{(i)}$ and $E^{(i)}$ greater than one or where two exceptional divisors and $C^{(i)}$ meet.

Definition 2.1. (i) $X_{k} \stackrel{\pi_{k}}{\rightarrow} \ldots \stackrel{\pi_{1}}{\rightarrow} X_{1} \rightarrow \mathbb{C}^{2}$ is called a standard resolution of $(C, 0)$ if all branches of $C^{(k)}$ are smooth, do not intersect each other, do intersect just one component of $E^{(k)}$ and do intersect this component transversally. (ii) For some i and k assume that $C_{i}^{(j)} \cap C_{k}^{(j)} \neq \phi$ but $C_{i}^{(j+1)} \cap C_{k}^{(j+1)}=\phi$ then $\gamma\left(C_{i}, C_{k}\right):=j+1$ is called the contact number of the branches $C_{i}$ and $C_{k}$.

We consider the following weighted graph, the resolution graph of $C$.

(i) To each component of $E^{(k)}$ a point $\bullet$ is associated.

(ii) To each component of $C^{(k)}$ a point $*$ is associated.

(iii) Two points are connected by an edge if the corresponding components intersect.

(iv) The points of type(i) are weighted. Let $E$ be a component of $E^{(k)}$.We give to the corresponding point the weight $i$ if $E$ is created in the $i-t h$ level of the blowing ups that is $i$ is minimal such that $\pi_{i+1} \circ \ldots \circ \pi_{n}(E)$ is not a point.

The multiplicity sequence corresponding to the strict transform of $C$ is called strict multiplicity sequence and the multiplicity sequence corresponding to the total transform of $C$ is called total multiplicity sequence.

Now we want to recall the Hamburger-Noether expansion of an irreducible plane curve singularity. We assume that the curve has a singular point at 0 and $x=x(t), y=y(t)$ is a parametrization. $x(t), y(t) \in \mathbb{C}[[t]]$ and $\operatorname{dim}_{\mathbb{C}} \mathbb{C}[[t]] /<$ $x(t), y(t)><\infty$. We further assume that $\operatorname{ord}(x(t)) \leq \operatorname{ord}(y(t))$.

Definition 2.2. A Hamburger-Noether expansion is a set of expressions of type 
$y=a_{01} x+a_{02} x^{2}+\ldots+a_{0, h} x^{h}+x^{h} z_{1}$

$x=a_{12} z_{1}^{2}+\ldots+a_{1, h_{1}} z_{1}^{h_{1}}+z_{1}^{h_{1}} z_{2}$

$z_{r-2}=a_{r-1,2} z_{r-1}^{2}+\ldots+a_{r-1, h_{r-1}} z_{r-1}^{h_{r-1}}+z_{r-1}^{h_{r-1}} z_{r}$

$z_{r-1}=a_{r, 2} z_{r}^{2}+\ldots$

Where $a_{j i} \in \mathbb{C}, z_{j} \in \mathbb{C}[[t]]$

$1 \leq \operatorname{ord}\left(z_{r}\right)<\ldots<\operatorname{ord}\left(z_{1}\right)<\operatorname{ord}(x) \leq \operatorname{ord}(y)$

Next we would like to recall the properties of the intersection multiplicity of two irreducible plane curves.

Let $(C, 0)$ be given by the parametrization $x=x(t), y=y(t)$ and $(D, 0)=$ $(V(g), 0), g \in \mathbb{C}[[x, y]]$ then the intersection multiplicity is defined by

$$
(C, 0) \cdot(D, 0)=\operatorname{ord}(g(x(t), y(t))) .
$$

One can use the Hamburger-Noether expansion to compute the intersection multiplicity as follows:

Let

$$
\begin{gathered}
z_{j-1}=\sum_{i} a_{j i} z_{j}^{i}+z_{j}^{h_{j}} z_{j+1}, 0 \leq j \leq r \\
z_{j-1}^{(1)}=\sum_{i} a_{j i}^{(1)} z_{j}^{(1) i}+z_{j}^{(1) h_{j}^{(1)}} z_{j+1}^{(1)}, 0 \leq j \leq r^{(1)}
\end{gathered}
$$

Let $s$ be the greatest integer for which $h=h^{(1)}, h_{1}=h_{1}^{(1)}, \ldots, h_{s-1}=h_{s-1}^{(1)}$ and $a_{j k}=a_{j k}^{(1)}$ for $j<s$ and $k \leq h_{j}$. Let $i$ be the least index such that $a_{s i} \neq a_{s i}^{(1)}\left(i \leq h_{s}+1, i \leq h_{s}^{(1)}+1\right)$. Finally set

$$
S=\sum_{j=0}^{s} h_{j} \cdot n_{j} \cdot n_{j}^{(1)}
$$

where $n_{j}=\operatorname{ord}\left(z_{j}\right)$ and $n_{j}^{(1)}=\operatorname{ord}\left(z_{j}^{(1)}\right)$.

Then we have: 
(i) if $i \leq h_{s}$ and $i \leq h_{s}^{(1)}$

$$
(C, D)=S+i \cdot n_{s} \cdot n_{s}^{(1)}
$$

(ii) if $i=h_{s}^{(1)}+1$

$$
(C, D)=S+h_{s} \cdot n_{s} \cdot n_{s}^{(1)}+n_{s+1}^{(1)} \cdot n_{s}
$$

(iii) if $i=h_{s}+1$

$$
(C, D)=S+h_{s}^{(1)} \cdot n_{s}^{(1)} \cdot n_{s}+n_{s+1} \cdot n_{s}^{(1)}
$$

Definition 2.3. Let $C$ be a reduced curve singularity then its milnor number is defined as $\mu(C)=\operatorname{dim} \mathbb{C}[[x, y]] /\left(\frac{\partial f}{\partial x}, \frac{\partial f}{\partial y}\right)$.

The aim of this paper is to reduce the computation of the resolution graph, the multiplicity sequences and Milnor number of any plane curve singularity to that of the irreducible branches. This can be done because of the following proposition.

Proposition 2.4. Let $(C, 0)=\cup_{i=1}^{r}\left(C_{i}, 0\right)$ be a plane curve singularity. Then the following data determine each other:

(i) The resolution graph of $C$.

(ii) The resolution graph of branches $\left(C_{i}, 0\right)$ together with the contact numbers $\gamma\left(C_{i}, C_{j}\right)$ for $i \neq j$.

To compute the contact numbers we use the following proposition.

Proposition 2.5. Let $(C, 0)$ respectively $(D, 0)$ be irreducible plane curve singularities with multiplicity sequences $\left(m_{0}, \ldots, m_{g}\right)$ respectively $\left(n_{0}, \ldots, n_{h}\right)$.For $k \geq g+1$ define $m_{k}=1$ and similarly for $k \geq h+1$ define $n_{k}=1$ then

$$
(C, 0) \cdot(D, 0)=\sum_{i=0}^{\gamma(C, D)} m_{i} \cdot n_{i}
$$

The proof of above two propositions can be found in book [5].

Proposition 2.6. Let $\mu(C)$ be the milnor number of a reduced germ $C$. If $\left(C_{i}\right)_{i=1 \ldots k}$ is decomposition of $C$. Then

$$
\mu(C)=\sum_{i=1}^{k} \mu\left(C_{i}\right)+2 \sum_{1 \leq i<j \leq k}\left(C_{i}, C_{j}\right)-k+1
$$

This proposition can be found in [3]. 


\section{The Algorithms}

In this section we present the algorithm to compute the incidence matrix of resolution graph, total multiplicities, strict multiplicities and Milnor number of $f$ by applying the results of section- 2 .

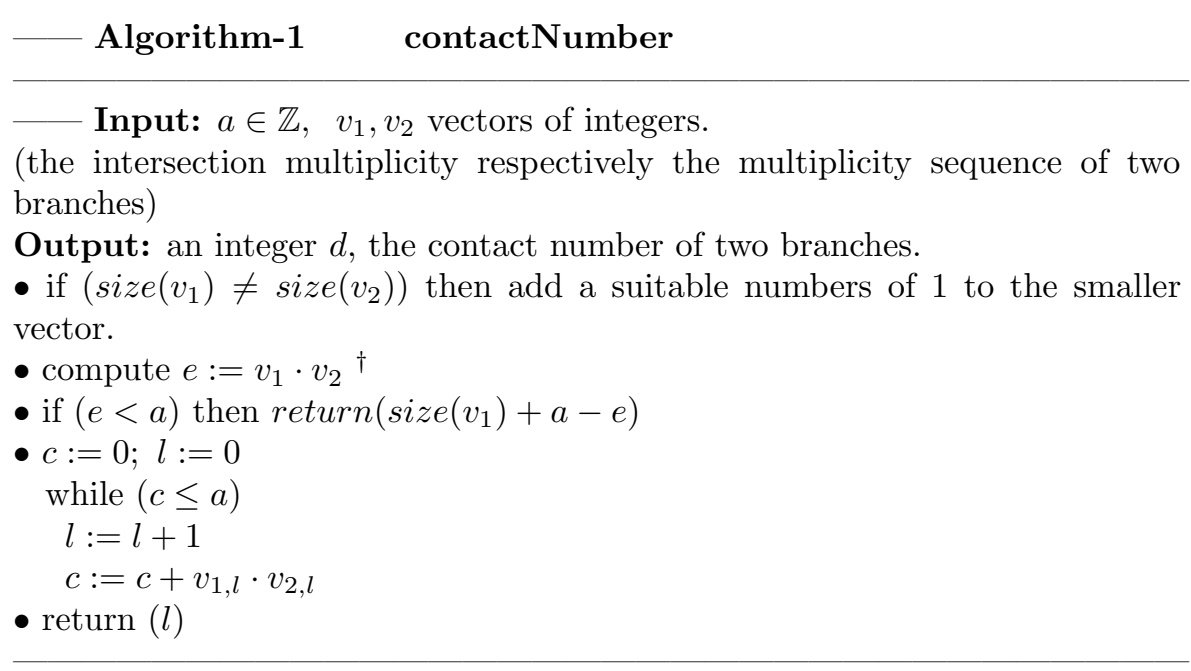

\section{Algorithm-2 resGraph}

Input: a polynomial $f,(f$ defines a reduced curve singularity $)$

Output: list $L$ containing the incidence matrix of the resolution graph, the total multiplicities, the strict multiplicities and the Milnor number of $f$

- factorize $f=f_{1}, \ldots, f_{r}$

- compute a list $H$, the Hamburger-Noether expansion ${ }^{\ddagger}$ for all $f_{i}$

- compute the matrix of intersection multiplicities ${ }^{\S}$ corresponding to the irre- $^{-}$ ducible branches of $f$

- compute the Milnor number $m$ by using the Milnor number of the branches and the intersection multiplicities.

- compute a list of invariants $\$$, the characteristic exponents and the multiplic-

\footnotetext{
$\dagger v_{1} \cdot v_{2}$ is the scalar multiplication of $v_{1}$ and $v_{2}$

$\ddagger$ In SINGULAR this can be done by using the procedure hnexpansion $\left(f_{i}\right)$ from hnoether.lib .

$\S_{\text {if }} h_{1}, h_{2}$ is the output of hnexpansion then in SINGULAR the procedure intersection $\left(h_{1}, h_{2}\right)$ computes the intersection multiplicity of the branches corresponding to $h_{1}$ and $h_{2}$.

If $h$ is the output of hnexpansion then in SINGULAR the procedure invariants $(h)$ gives a list of invariants corresponding to each branch, the first entry of the list contains
} 
ity sequence for each $h \in H$

- compute the matrix of contact numbers corresponding to the irreducible branches of $f$ (Algorithm-1)

- compute a list ${ }^{\|} L$ of the incidence matrix of the resolution graph, the total multiplicities and the strict multiplicities of $f$

- add the Milnor number to the list L

- $\operatorname{return}(L)$

\section{Timings and Table}

In this section we provide some examples and a table which gives a time comparison between the algorithm totalmultiplicities, algorithm milnor and the algorithm resGraph.Timings are conducted by using Singular 3-1-3 on an Intel@T2400, dual core $1.83 \mathrm{GHz}$ processor, 1 GB RAM under the Window 2007 operating system.

We consider the following polynomials:

$$
\begin{aligned}
f_{1}= & \left(\left(x^{7}-y^{6}\right)^{4}-x^{20} y^{10}+x^{35}\right) \cdot\left(\left(x^{7}-y^{6}\right)^{3}-x^{24}+y^{25}\right) \cdot\left(\left(x^{7}-y^{5}\right)^{3}-x^{24}-y^{25}\right) \\
f_{2}= & \left(x^{14}+x^{5} y^{5}+y^{10}+y^{15}\right) \cdot\left(x^{16}+x^{18}+y^{20}+y^{23}\right) \\
f_{3}= & \left(x^{14}-y^{12}+x^{10} y^{4}+y^{19}\right) \cdot\left(x^{12}-y^{14}+x^{15}+y^{15}\right) \cdot\left(\left(x^{6}-y^{5}\right)^{4}-x^{26} y^{12}+\right. \\
& \left.x^{28}+y^{30}\right) \cdot\left(x^{14}+y^{18}+y^{21}\right) \\
f_{4}= & \left(x^{8}+2 y^{14}\right) \cdot\left(x^{10}+5 y^{10}\right) \cdot\left(y^{2}-x^{3}\right) \cdot\left(x^{2}-y^{3}\right) \\
f_{5}= & \left(x^{18}+y^{24}+x^{29}\right) \cdot\left(x^{14}+y^{18}+y^{21}\right) \cdot\left(x^{9}-x^{3} y^{3}+y^{11}\right) \cdot\left(x^{15}-y^{10}-y^{19}\right) \\
f_{6}= & \left(x^{16}+3 y^{18}\right) \cdot\left(x^{5}+7 y^{5}\right) \\
f_{7}= & \left(\left(x^{7}-y^{6}\right)^{4}-x^{20} y^{10}+x^{35}\right) \cdot\left(\left(x^{7}-y^{6}\right)^{3}-x^{24}+y^{25}\right) \\
f_{8}= & \left(x^{18}+y^{24}+x^{29}\right) \cdot\left(x^{14}+y^{18}+y^{21}\right) \cdot\left(x^{9}-x^{3} y^{3}+y^{11}\right)
\end{aligned}
$$

the characteristics exponents, semi-group generators, the Puiseux pairs, the degree of the conductor, the sequence of multiplicities and the second entry of the list contains the contact matrix of the branches, the intersection multiplicities of the branches and the delta invariant of $f$.

"If $N$ is the contact matrix and $A$ is the list of characteristic exponents of all branches of $f$ then in the SINGULAR procedure totalmultiplicities $(N, A)$ from alexpoly.lib gives a list of the incidence matrix of the resolution graph, the total multiplicities and the strict multiplicities of $f$. 


\begin{tabular}{|c|c|c|c|}
\hline Poly & Timing(totalmultiplicities) & Timing(resGraph) & Timing(milnor) \\
\hline$f_{1}$ & $27 \mathrm{~min}$ & $2 \mathrm{sec}$ & not enough memory \\
\hline$f_{2}$ & $40 \mathrm{~min}$ & $2 \mathrm{sec}$ & $2 \mathrm{sec}$ \\
\hline$f_{3}$ & $55 \mathrm{~min}$ & $3 \mathrm{sec}$ & $1 \mathrm{~min}$ \\
\hline$f_{4}$ & $>1 \mathrm{hr}$ & $3 \mathrm{~min}$ & $2 \mathrm{sec}$ \\
\hline$f_{5}$ & $>1 \mathrm{hr}$ & $3 \mathrm{sec}$ & not enough memory \\
\hline$f_{6}$ & not enough memory & $10 \mathrm{sec}$ & $2 \mathrm{sec}$ \\
\hline$f_{7}$ & $1 \mathrm{~min}$ & $2 \mathrm{sec}$ & $6 \mathrm{~min}$ \\
\hline$f_{8}$ & $4 \mathrm{~min}$ & $2 \mathrm{sec}$ & $8 \mathrm{~min}$ \\
\hline
\end{tabular}

\section{References}

[1] Campillo,A.: Algebroid Curves in Positive Characteristics.Lecture Notes in Mathematics 813,Springer(1980).

[2] Decker, W.; Greuel, G.-M.; Pfister, G.; Schönemann, H.: SinguLAR 3-1-1 - A computer algebra system for polynomial computations. http://www.singular.uni-kl.de (2010).

[3] Evelia, R.; Garcia, B.; Andrzej, L.; Arkadiusz, P.: Characterization of non-degenerate plane curve singularities.

[4] Greuel, G.-M.; Pfister, G.: A Singular Introduction to Commutative Algebra. Second edition, Springer (2007).

[5] De Jong, T.; Pfister, G.: Local Analytic Geometry. Vieweg (2000).

Muhammad Ahsan Binyamin,

Department of Applied Sciences, National Textile University,

Faisalabad, 38000 Pakistan Email: ahsanbanyamin@gmail.com 